\title{
The Risk Factors and Predictive Nomogram for Length Of Stay More than 14 Days in Undergoing TKA Patients: A Secondary Analysis Based on a Cohort Study in Singapore
}

Bo Liu

The Affiliated Hospital of Qingdao University

\section{Yijiang Ma}

The Affiliated Hospital of Qingdao University

Chunxiao Zhou

The Affiliated Hospital of Qingdao University zhijie wang ( $\nabla$ simonwang1969@163.com)

The Affiliated Hospital of Qingdao University https://orcid.org/0000-0002-4944-1750

\section{Research Article}

Keywords: total knee arthroplasty, length of stay, the risk factor, nomogram, secondary analysis, a cohort study

Posted Date: November 8th, 2021

DOI: https://doi.org/10.21203/rs.3.rs-1041995/v1

License: (9) This work is licensed under a Creative Commons Attribution 4.0 International License. Read Full License 


\section{Abstract}

\section{Objective:}

The aim was to explore the risk factors and establish a predictive nomogram of length of stay (LOS) more than 14 days in a undergoing Total knee arthroplasty (TKA) cohort .

\section{Methods:}

We used the raw data, a retrospective cohort study of 2622 patients undergoing TKA in Singapore, for secondary analysis. The LASSO regression was used to optimize feature selection for the LOS more than 14 days. The Multivariable logistic regression analysis was applied to build a predicting nomogram by using the feature selected in the LASSO regression model. In order to evaluate the prediction ability of the model, we calculated the C-index. Simultaneously, the ROC curve, the Calibration curve and the DCA curve was draw to assess the model. Finally, we used 1000 times bootstrap method to verify the accuracy of the model.

\section{Results:}

Finally, 100(3.81\%) patients were hospitalized for more than 14 days and 2522 patients $(96.19 \%)$ were less 14 days. Patient age, ASA status, type of anesthesia, operation duration, procedure description, DM, IHD, CHF, day of operation and blood transfusion were determined and incorporated into the diagnostic nomogram. The C-index was $0.797(95 \% \mathrm{Cl}$ : 0.755-0.839). The calibration curve showed that the model had good recognition ability.The DCA curve analysis showed that the risk nomogram of length of stay more than 14 days was clinically useful.The $\mathrm{C}$-index is 0.763 through 1000 times bootstrapping validation.

\section{Conclusion:}

We used the age, ASA status, type of anesthesia, operation duration, procedure description, DM, IHD, CHF, day of operation and blood transfusion to establish the clinical prediction model, this method can conveniently predict the risk of individual patients with total knee arthroplasty length of stay for more than 14 days.

\section{Introduction}

Total knee arthroplasty (TKA) is one of the most common orthopedic surgeries, Studies have shown that over time, the prevalence rate has increased significantly and shifted to a younger age, and the prevalence rate in women is higher than that in men ${ }^{1}$.The TKA was mainly used for the treatment of pain, mobility limitation and joint deformity caused by osteoarthritis, rheumatoid arthritis and knee trauma ${ }^{2}$. It can help patients restore knee joint function and significantly improve the quality of life of patients ${ }^{3}$.The increased life expectancy and the aging population have led to a dramatic increase in the number of patients with knee disorders, and total knee arthroplasty (TKA) is currently the most important and 
effective treatment for severe knee disorders, the demand for TKA will increase dramatically in the coming years ${ }^{4,5}$. It is estimated that more than 700,000 TKA surgeries are performed in the United States every year, and this number will increase to nearly 3.5 million by 2030 . Therefore, it is inevitable to perform TKA in an economical and effective way ${ }^{6}$.

The associated costs of surgical procedures, inpatient care, rehabilitation, and postoperative care have increased substantially due to enhanced intra - and perioperative management ${ }^{7}$. Meanwhile, as the epidemic of new coronary pneumonia spread, there was an obvious decline in the global economy, and the Medical insurance expenditure increased significantly. Some countries have removed the TKA from the List of Medicare Outpatient Payment System Rules ${ }^{8}$. The focus of reducing hospitalization cost is to reduce Length Of Stay(LOS). The shortening LOS will increase the turnover rate of inpatients, reduce medical costs and reduce the medical burden of the society ${ }^{9}$.

In addition, TKA surgery may be accompanied by serious complications, which will adversely affect the prognosis of patients and increase the possibility of disability and death ${ }^{10}$. However, the longer LOS is a risk factor for the postoperative complications. It is particularly important to reduce postoperative complications such as postoperative infection, thromboembolism, postoperative delirium and cognitive dysfunction ${ }^{11}$. Therefore, how to determine the factors of LOS , reduce the incidence of surgical complications, decress hospitalization expenses and save medical resources are the problems,which should be solved by the doctors. The purpose of this study was to explore the risk factors and establish a predictive nomogram of length of stay (LOS) more than 14 days in a undergoing Total knee arthroplasty (TKA) .

\section{Patients And Methods}

\section{Patients Dates}

Our study is a secondary analysis based on a single-center retrospective cohort study in Singapore. Patients who underwent TKA at Singapore General Hospital from January 2013 to June 2014 were selected $^{12}$. We download the raw data uploaded by Abdullah et al at the“DATADRYAD”web site (www.datadryad.org). Abdullah et al have authorized the ownership of the original data to the datadryad Web site; therefore, we could utilize these data for secondary analysis on a different hypothesis without violating the authors'rights.

The study was approved by the Ethics Committee of the Affiliated Hospital of Qingdao University. The Ethics Committee particularly approved that informed consent was not required because of being approved by the“DATADRYAD"Web site (www.datadryad.org) and data were analyzed anonymously.The ethics committee waived the requirement for informed consent from all patients.

\section{Statistical analysis}


Statistical analysis was performed using R software (version 3.1.1; https://www.R-project.org). Chisquared tests (categorical variables) and one-way analysis of variance or $\mathrm{K}-\mathrm{W}$ test (continuous variables of a normal distribution or skewed distribution) were appropriately performed to verify significant differences between different groups.

We used R package "glmnet" for doing lasso regression analysis. Lasso regression can perform variable screening and complexity adjustment while fitting the generalized linear model. Variable screening refers to not putting all variables into the model for fitting, but selectively putting variables into the model to get better performance parameters. Complexity adjustment is to avoid over fitting by controlling the complexity of the model through a series of parameters.Finally, we got the result of lasso's regression. All potential predictors were applied to develop a cohort model that predicted the risk of LOS more than 14 days.

Multivariate Logistic regression analysis was used to establish the prediction model combining the minimum absolute contraction and the selected features in the selection operator regression model. The accuracy of the prediction model was evaluated by the ROC curve. A decision curve analysis was performed by quantifying the net benefit at different threshold probabilities in the TKA cohort to determine the clinical usefulness of LOS more than 14 days risk nomogram. Furthermore, the calibration curves and decision curve analysis (DCA) curves were established for the assessment of the nomograms.

C-index, which is the consistency index, is used to evaluate the prediction ability of the model. We use 1000 bootstrap times to verify the accuracy of the model. There is a random sample put back in the original data, and the sample size remains unchanged. The obtained sample is called bootstrap sample.

\section{Results}

\section{The Demographic characteristics of patients}

Finally, 2622 patients who underwent TKA from January 2013 to June 2014,a retrospective cohort, were included in the study. 2622 patients(96.19\%) were hospitalized for less than 14 days, however, 100 patients(3.81\%) were hospitalized for more than 14 days.In Table 1, the proportion of patients with advanced age ( $\geq 70$ years) was higher in the prolonged LOS group than in patients with normal LOS ( $<70$ years), and the difference was statistically significant $(P \leq 0.001)$. There were no significant differences in $\mathrm{BMI}$, race, and sex distribution between the 2 groups. However, patients in the LOS extended group had a high ASA score $(p \leq 0.001)$, a long operation time $(p \leq 0.001)$, a high ratio of bilateral knee replacement $(p \leq 0.001)$, a high incidence of anemia and diabetes ( $p \leq 0.001)$, a history of cough ( $p \leq 0.001)$, a high risk of transfusion during operation $(p \leq 0.001)$, repeated operation $(p \leq 0.001)$ during hospitalization, and general anesthesia $(p \leq 0.001)$.

\section{The Feature Risk Foctors Selection}


This study was based on data on demographic, disease, and treatment characteristics of 2622 patients undergoing knee replacement in the cohort. 17 features were reduced to ten potential predictors on TKA patients in the cohort (Figure1 A and B) and were with nonzero coefficients in the LASSO regression model, as shown in Figure 1.These characteristics included Age, ASA Status, type of anaesthesia, operation duration, procedure description, DM, IHD, CHF, day of operation and blood transfusion (Table 2).

\section{The Development of Nomogram for LOS More Than 14 Days}

We developed a model containing the above 10 independent predictors and expressed them as a nomogram ( Figure2).

\section{The Validation of Prediction Nomogram}

The ROC curve shows that the rosette has high predictive performance, and its AUC is 0.797 (Figure3).This shows that the prediction model has strong prediction ability. The calibration curves used to predict LOS more than 14 days risk nomogram in knee patients in this study cohort showed good consistency (Figure4). Similarly, the calibration curve also shows that the prediction ability of the model is strong.

To quantify the discrimination performance of the LOA more than 14 days nomogram, C-index was measured. The $\mathrm{C}$-index for the prediction nomogram was 0.797 (95\% Cl: $0.755-0.839)$ for the cohort.The C-index is 0.763 through 1000 times bootstrapping validation. The DCA of LOS more than 14 days indicated that this nomogram can serve as an excellent diagnostic tool for patients undergoing TKA(Figure 5).

\section{Discussion}

The aim of our study was to develop and validate a LOS more than 14 days risk prediction model for TKA patients. The risk factors were Age, ASA status, type of anesthesia, operation duration, procedure description, DM, IHD, CHF, day of operation and blood transfusion.Ultimately, We built the nomogram using the above risk factors for LOS more than 14 days in TKA patients. The Nomogram transforms the complex regression equation into a visual graph, which makes the results of the prediction model more readable and convenient for patient evaluation. Because of the intuitive and easy to understand characteristic of nomogram, it has gradually been paid more and more attention and applied in medical research and clinical practice.

In the risk prediction nomogram with LOS more than 14 days in this study, the highest independent predictor score was blood transfusion, many studies have come to the same conclusion, and the transfusion rate was directly related to $\operatorname{LOS}^{13-15}$. Autologous blood transfusion is often required when the 
blood loss is between 1000-1500 ml. research shows that the total blood loss after TKA may be as high as $2000 \mathrm{ml}$, and the proportion of blood transfusion may be as high as $67 \%{ }^{16,17}$. In a cohort of 228,316 TKA patients at 922 hospitals, the mean predicted probability of TKA transfusion was $7.9 \%$, with $60 \%$ (95\% Cl, 36\%-87\%) patients with LOS more than 3 days $^{13}$. In a cross-sectional study of 4,544,999 patients who received TKA between January 2000 and December 2009, blood transfusion were associated inhospital mortality, LOS increased by $0.71 \pm 0.01$ days ${ }^{14}$. In addition, Danninger et al. ${ }^{15}$ showed a significantly higher rate of major complications in patients receiving transfusion $(19.1 \%$ vs $11.2 \%$, $P<0.0001)$, the mean length of hospital stay was significantly increased.

Our study found that the date of operation was a major factor in predicting the risk of LOS more than 14 days after blood transfusion. Similarly, studies demonstrated that operation procedure or factors related to doctors-nurses provide clinically relevant improvement in explaining length of stay in addition to patient-related risk factors ${ }^{8}$. In a prospective cohort study of 4,509 patients who underwent initial TKA at four hospitals between January 1, 2016 and September 30, 2017, surgery later in the day predicted a longer hospital stay, with patients who had surgery on Friday having a significantly longer LOS than patients who had surgery on Monday ${ }^{8}$. Our study showed that the risk prediction scores of operations on Monday, Tuesday and Friday were much higher than those on Thursday. The length of hospital stay was related to the date of operation. Possible reasons for this result include patients from different countries, surgical hospital system, doctors' mood and doctors' preferences.

This study found that the greater the variety of comorbid diseases, that is, the higher the comorbidity index, and the longer the hospital stay. Swain et al. ${ }^{18}$ showed that $67 \%$ of patients with osteoarthritis had at least one other chronic condition, $20 \%$ more than those without osteoarthritis. The results of this study suggest that co-morbidity CHF, DM, and IHD are independent predictors of LOS more than 14 days. Similarly, some previous studies suggested that the TKA patients with comorbidities had prolonged LOS $^{19-23}$. Higuera et al. ${ }^{22}$ showed that chronic heart failure was associated with longer hospital stays and increased rates of major postoperative complications in TKA patients. In a study of 15,321 TKA patients, $18.2 \%$ had a medical comorbidity DM, with a $300 \%$ increase in overall mortality. Belmont et al. ${ }^{23}$ found that DM was an independent predictor of hospitalization of 4 days or more.

ASA status was also particularly important for predicting LOS more than 14 days of risk. Consistent with previous literature, ASA-status more than 3 is an independent risk factor for prolonged LOS and increased postoperative complications ${ }^{23,24}$. The predicted risk score of bilateral total knee arthroplasty is higher than that of unilateral TKA, probably because bilateral patients suffer more blood loss, more severe hypotension, and more cardiac, respiratory, and neurological complications than unilateral patients ${ }^{25,26}$. In this study, it was suggested that patients undergoing knee revision were also predictors of LOS more than 14 days of risk, and that revision arthroplasty was associated with longer hospital stays and higher rates of complications and mortality, similar to results confirmed in previous studies ${ }^{27,28}$. 
The type of anesthesia also played an important role in predicting the risk of LOS more than 14 days. In both primary and revised TKA patients, general anesthesia was associated with an increased risk of postoperative complications, such as thromboembolism and surgical incision infection, as well as increased the LOS, blood transfusion rates, postoperative opioid consumption, and surgical time, compared with epidural or spinal anesthesia ${ }^{29-33}$.In addition, age and operation duration were independent predictors of LOS more than 14 days risk, similar to previous finding ${ }^{23,34}$. Limitations of this study include: as the included cases excluded patients with GA combined with RA and other anesthesia methods, the results of this study cannot be used to predict the risk of this population.

There are still some limitations in our study. Firstly, this study is a retrospective study, using clinical data from a single center. Therefore, there may be differences in treatment strategies, race, and so on. Second, because of differences in healthcare Settings and practices, predictive models developed in one country are unlikely to be directly applicable in another country, requiring external validation and updating of the predictive performance of models in other new patient. Finally, there may be some influencing factors not included in this paper, including patient income, medical expenses, medical insurance, hospital location, etc. Potential factors not included may also have some influence on the results.

\section{Conclusion}

We based on the variables, including age, ASA status, type of anesthesia, operation duration, procedure description, DM, IHD, CHF, day of operation and blood transfusion, established the risk prediction model of LOS more than 14 days in undergoing TKA patients. We found that the model had good predictive ability. Clinicians can use the model for hospitalized patients to reduce the length of hospital stay, reduce complications, and save medical resources.

\section{Abbreviations}

LOS: length of stay; TKA:total knee arthroplasty;ROC: receiver operating characteristic; OR: odds ratio; AUC: area under the curve; Cl: confidence interval;DM:diabetes mellitus; IHD:ischemic heart disease; CHF:Congestive Hearts Failure;LASSO, least absolute shrinkage and selection operator; SE, standard error.

\section{Declarations}

\section{Data availability statement}

Availability of Data and Materials: Data can be down-loaded from "DATADRYAD" database. Dryad data package:Abdullah HR, Sim E, Hao Y, Lin G, Liew GHC, Lamoureux EL,Tan MH (2017). Data from: Association between preoperative anemia with length of hospital stay among patients undergoing primary total knee arthroplasty in Singapore:a single-center retrospective study. Dryad Digital Repository. https://doi.org/10.5061/dryad.73250.

\section{Funding}


This study was financially supported by the Department of Science Technology of Shandong Province for

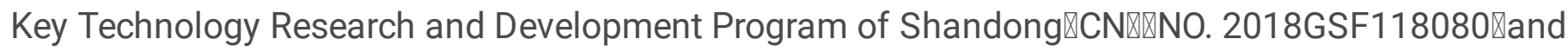
Qingdao Municipal Science and Technology Bureau for demotic science ar technology program $\otimes$ NO. 19-61-16-nsh $\llbracket$

\section{Author contributions}

$\mathrm{BL}$ performed the data analysis. $\mathrm{BL}, \mathrm{CX} Z$ wrote the manuscript. $\mathrm{BL}, \mathrm{CX}$ Zand $\mathrm{YJ}$ M contributed to the manuscript revise. YJ M contributed to literature search and data extraction. BL,CX Z and ZJ W conceived and designed the study. All authors have read and approved the final version of the manuscript.

\section{Conflict of interest}

The authors declare that the research was conducted in the absence of any commercial or financial relationships that could be construed as a potential conflict of interest.

\section{Ethics approval and consent to participate}

The study was approved by the Ethics Committee of the Affiliated Hospital of Qingdao University. The Ethics Committee particularly approved that informed consent was not required because of being approved by the DRYAD and data were analyzed anonymously. The ethics committee waived the requirement for informed consent from all patients.

\section{Consent for publication}

Not applicable.

\section{Competing interests}

The authors declare that they have no competing interests.

\section{References}

1. Maradit Kremers, H. et al. Prevalence of Total Hip and Knee Replacement in the United States. J Bone Joint Surg Am 97, 1386-1397, doi:10.2106/jbjs.N.01141 (2015).

2. Ji, X. \& Ke, W. Two types of anaesthesia and length of hospital stay in patients undergoing unilateral total knee arthroplasty (TKA): a secondary analysis based on a single-centre retrospective cohort study in Singapore. BMC Anesthesiol 21, 242, doi:10.1186/s12871-021-01459-7 (2021).

3. Ethgen, O., Bruyère, O., Richy, F., Dardennes, C. \& Reginster, J. Y. Health-related quality of life in total hip and total knee arthroplasty. A qualitative and systematic review of the literature. J Bone Joint Surg Am 86, 963-974, doi:10.2106/00004623-200405000-00012 (2004).

4. Kim, S. H. Morbid obesity and excessive hospital resource consumption for unilateral primary hip and knee arthroplasty. J Arthroplasty 25, 1258-1266, doi:10.1016/j.arth.2009.09.005 (2010). 
5. Losina, E., Thornhill, T. S., Rome, B. N., Wright, J. \& Katz, J. N. The dramatic increase in total knee replacement utilization rates in the United States cannot be fully explained by growth in population size and the obesity epidemic. J Bone Joint Surg Am 94, 201-207, doi:10.2106/jbjs.J.01958 (2012).

6. Kurtz, S., Ong, K., Lau, E., Mowat, F. \& Halpern, M. Projections of primary and revision hip and knee arthroplasty in the United States from 2005 to 2030. J Bone Joint Surg Am 89, 780-785, doi:10.2106/jbjs.F.00222 (2007).

7. Belatti, D. A., Pugely, A. J., Phisitkul, P., Amendola, A. \& Callaghan, J. J. Total joint arthroplasty: trends in medicare reimbursement and implant prices. J Arthroplasty 29, 1539-1544, doi:10.1016/j.arth.2014.03.015 (2014).

8. Cleveland Clinic Orthopaedic Arthroplasty, G. The Main Predictors of Length of Stay After Total Knee Arthroplasty: Patient-Related or Procedure-Related Risk Factors. J Bone Joint Surg Am 101, 10931101, doi:10.2106/JBJS.18.00758 (2019).

9. Molloy, I. B., Martin, B. I., Moschetti, W. E. \& Jevsevar, D. S. Effects of the Length of Stay on the Cost of Total Knee and Total Hip Arthroplasty from 2002 to 2013. J Bone Joint Surg Am 99, 402-407, doi:10.2106/JBJS.16.00019 (2017).

10. Parvizi, J. et al. Total joint arthroplasty: When do fatal or near-fatal complications occur? J Bone Joint Surg Am 89, 27-32, doi:10.2106/jbjs.E.01443 (2007).

11. Kehlet, H. Fast-track hip and knee arthroplasty. The Lancet 381, 1600-1602, doi:10.1016/s01406736(13)61003-x (2013).

12. Abdullah, H. R. et al. Association between preoperative anaemia with length of hospital stay among patients undergoing primary total knee arthroplasty in Singapore: a single-centre retrospective study. BMJ Open 7, e016403, doi:10.1136/bmjopen-2017-016403 (2017).

13. Menendez, M. E. et al. Variation in Use of Blood Transfusion in Primary Total Hip and Knee Arthroplasties. J Arthroplasty 31, 2757-2763 e2752, doi:10.1016/j.arth.2016.05.022 (2016).

14. Klika, A. K. et al. Primary Total Knee Arthroplasty Allogenic Transfusion Trends, Length of Stay, and Complications: Nationwide Inpatient Sample 2000-2009. The Journal of Arthroplasty 29, 20702077, doi:10.1016/j.arth.2014.06.018 (2014).

15. Danninger, T. et al. Blood transfusions in total hip and knee arthroplasty: an analysis of outcomes. ScientificWorldJournal 2014, 623460, doi:10.1155/2014/623460 (2014).

16. Rosencher, N. et al. Orthopedic Surgery Transfusion Hemoglobin European Overview (OSTHEO) study: blood management in elective knee and hip arthroplasty in Europe. Transfusion 43, 459-469, doi:10.1046/j.1537-2995.2003.00348.x (2003).

17. Bong, M. R. et al. Risks associated with blood transfusion after total knee arthroplasty. J Arthroplasty 19, 281-287, doi:10.1016/j.arth.2003.10.013 (2004).

18. Swain, S., Sarmanova, A., Coupland, C., Doherty, M. \& Zhang, W. Comorbidities in Osteoarthritis: A Systematic Review and Meta-Analysis of Observational Studies. Arthritis care \& research 72, 9911000, doi:10.1002/acr.24008 (2020).

19. <A prediction model for length of stay.pdf>. doi:10.1302/0301-620X.95B11. 
20. Jans, Ø., Jørgensen, C., Kehlet, H. \& Johansson, P. I. Role of preoperative anemia for risk of transfusion and postoperative morbidity in fast-track hip and knee arthroplasty. Transfusion 54, 717726, doi:10.1111/trf.12332 (2014).

21. Mathijssen, N. M., Verburg, H., van Leeuwen, C. C., Molenaar, T. L. \& Hannink, G. Factors influencing length of hospital stay after primary total knee arthroplasty in a fast-track setting. Knee Surg Sports Traumatol Arthrosc 24, 2692-2696, doi:10.1007/s00167-015-3932-x (2016).

22. Higuera, C. A., Elsharkawy, K., Klika, A. K., Brocone, M. \& Barsoum, W. K. 2010 Mid-America Orthopaedic Association Physician in Training Award: predictors of early adverse outcomes after knee and hip arthroplasty in geriatric patients. Clin Orthop Relat Res 469, 1391-1400, doi:10.1007/s11999-011-1804-3 (2011).

23. Belmont, P. J., Jr., Goodman, G. P., Waterman, B. R., Bader, J. O. \& Schoenfeld, A. J. Thirty-day postoperative complications and mortality following total knee arthroplasty: incidence and risk factors among a national sample of 15,321 patients. J Bone Joint Surg Am 96, 20-26, doi:10.2106/JBJS.M.00018 (2014).

24. Gromov, K., Troelsen, A., Stahl Otte, K., Ørsnes, T. \& Husted, H. Morbidity and mortality after bilateral simultaneous total knee arthroplasty in a fast-track setting. Acta orthopaedica 87, 286-290, doi:10.3109/17453674.2016.1141631 (2016).

25. Lynch, N. M., Trousdale, R. T. \& Ilstrup, D. M. Complications after concomitant bilateral total knee arthroplasty in elderly patients. Mayo Clin Proc 72, 799-805, doi:10.4065/72.9.799 (1997).

26. Parvizi, J., Sullivan, T. A., Trousdale, R. T. \& Lewallen, D. G. Thirty-day mortality after total knee arthroplasty. J Bone Joint Surg Am 83, 1157-1161, doi:10.2106/00004623-200108000-00004 (2001).

27. Mahomed, N. N. et al. Rates and outcomes of primary and revision total hip replacement in the United States medicare population. J Bone Joint Surg Am 85, 27-32, doi:10.2106/00004623200301000-00005 (2003).

28. Pulido, L. et al. In hospital complications after total joint arthroplasty. J Arthroplasty 23, 139-145, doi:10.1016/j.arth.2008.05.011 (2008).

29. Wilson, J. M., Farley, K. X., Erens, G. A. \& Guild, G. N., 3rd. General vs Spinal Anesthesia for Revision Total Knee Arthroplasty: Do Complication Rates Differ? J Arthroplasty 34, 1417-1422, doi:10.1016/j.arth.2019.03.048 (2019).

30. Chang, C. C., Lin, H. C., Lin, H. W. \& Lin, H. C. Anesthetic management and surgical site infections in total hip or knee replacement: a population-based study. Anesthesiology 113, 279-284, doi:10.1097/ALN.0b013e3181e2c1c3 (2010).

31. Moucha, C. S., Weiser, M. C. \& Levin, E. J. Current Strategies in Anesthesia and Analgesia for Total Knee Arthroplasty. The Journal of the American Academy of Orthopaedic Surgeons 24, 60-73, doi:10.5435/jaaos-d-14-00259 (2016).

32. Basques, B. A., Toy, J. O., Bohl, D. D., Golinvaux, N. S. \& Grauer, J. N. General compared with spinal anesthesia for total hip arthroplasty. J Bone Joint Surg Am 97, 455-461, doi:10.2106/jbjs.N.00662 (2015). 
33. Pugely, A. J., Martin, C. T., Gao, Y., Mendoza-Lattes, S. \& Callaghan, J. J. Differences in short-term complications between spinal and general anesthesia for primary total knee arthroplasty. J Bone Joint Surg Am 95, 193-199, doi:10.2106/jbjs.K.01682 (2013).

34. Kuperman, E. F., Schweizer, M., Joy, P., Gu, X. \& Fang, M. M. The effects of advanced age on primary total knee arthroplasty: a meta-analysis and systematic review. BMC geriatrics 16,41 , doi:10.1186/s12877-016-0215-4 (2016).

\section{Tables}

\section{Table 1 Baseline characteristics of selected participants}


LOS more than 14 days?

Gender

Male

Female

Age(Years)

$\square 70$

$\geq 70$

Race

Chinese

Indian

Malay

Others

BMI

$\square 24$

$\geq 24$

ASA_Status

1

2

3

$\mathrm{Hb}(\mathrm{g} / \mathrm{dL})$

$\square 12$

$\geq 12$

Type_of_Anaesthesia

GA

RA

Operation_Duration

$\square 100$

$\geq 100$

Procedure_Description
No( $n=2522) \quad$ Yes $(n=100) \quad P$-value

0.966

$610(24.187 \%) \quad 24(24.000 \%)$

$1912(75.813 \%) \quad 76(76.000 \%)$

0.009

$1064(42.189 \%) \quad 29(29.000 \%)$

$1458(57.811 \%) \quad 71(71.000 \%)$

0.095

$2119(84.021 \%) \quad 87(87.000 \%)$

$146(5.789 \%) \quad 4(4.000 \%)$

$185(7.335 \%) \quad 3(3.000 \%)$

$72(2.855 \%) \quad 6(6.000 \%)$

0.396

$517(20.500 \%) \quad 24(24.000 \%)$

$2005(79.500 \%) \quad 76(76.000 \%)$

$<0.001$

$180(7.137 \%) \quad 4(4.000 \%)$

$2196(87.074 \%) \quad 78(78.000 \%)$

$146(5.789 \%) \quad 18(18.000 \%)$

0.003

$479(18.993 \%) \quad 31(31.000 \%)$

$2043(81.007 \%) \quad 69(69.000 \%)$

0.001

$909(36.043 \%) \quad 52(52.000 \%)$

$1613(63.957 \%) \quad 48(48.000 \%)$

$<0.001$

$1862(73.830 \%) \quad 58(58.000 \%)$

$660(26.170 \%) \quad 42(42.000 \%)$ 
Unilateral

Bilateral

Revision

Smoking

No

Yes

OSA

No

Yes

DM

No

Yes

IHD

No

Yes

CHF

No

Yes

CVA

No

Yes

which Day of doing operation in a week?

Mon

Tue

Wed

Thu

Fri

Sat

\begin{tabular}{ll}
$2314(91.753 \%)$ & $80(80.000 \%)$ \\
\hline $188(7.454 \%)$ & $18(18.000 \%)$ \\
\hline $20(0.793 \%)$ & $2(2.000 \%)$
\end{tabular}

0.873

$2283(90.523 \%) \quad 91(91.000 \%)$

$239(9.477 \%) \quad 9(9.000 \%)$

0.270

$\begin{array}{ll}2289(90.761 \%) & 94(94.000 \%) \\ 233(9.239 \%) & 6(6.000 \%)\end{array}$

0.012

\begin{tabular}{ll}
$2066(81.919 \%)$ & $72(72.000 \%)$ \\
\hline $456(18.081 \%)$ & $28(28.000 \%)$
\end{tabular}

0.022

$\begin{array}{ll}2399(95.123 \%) & 90(90.000 \%) \\ 123(4.877 \%) & 10(10.000 \%)\end{array}$

$<0.001$

2504 (99.286\%) $95(95.000 \%)$

$18(0.714 \%) \quad 5(5.000 \%)$

0.353

\begin{tabular}{ll}
$2478(98.255 \%)$ & $97(97.000 \%)$ \\
\hline $44(1.745 \%)$ & $3(3.000 \%)$
\end{tabular}

0.002

$410(16.257 \%) \quad 25(25.000 \%)$

$561(22.244 \%) \quad 28(28.000 \%)$

$435(17.248 \%) \quad 13(13.000 \%)$

$590(23.394 \%) \quad 10(10.000 \%)$

$395(15.662 \%) \quad 22(22.000 \%)$

$131(5.194 \%) \quad 2(2.000 \%)$ 


$\begin{array}{lll}\text { No } & 2402(95.242 \%) & 67(67.000 \%) \\ \text { Yes } & 120(4.758 \%) & 33(33.000 \%)\end{array}$

Results in the table: mean + SD / N (\%)

Table 2 The Risk factors of LASSO Regression and Multivariate logistic Regression for LOS More Than 14 Days

Figures
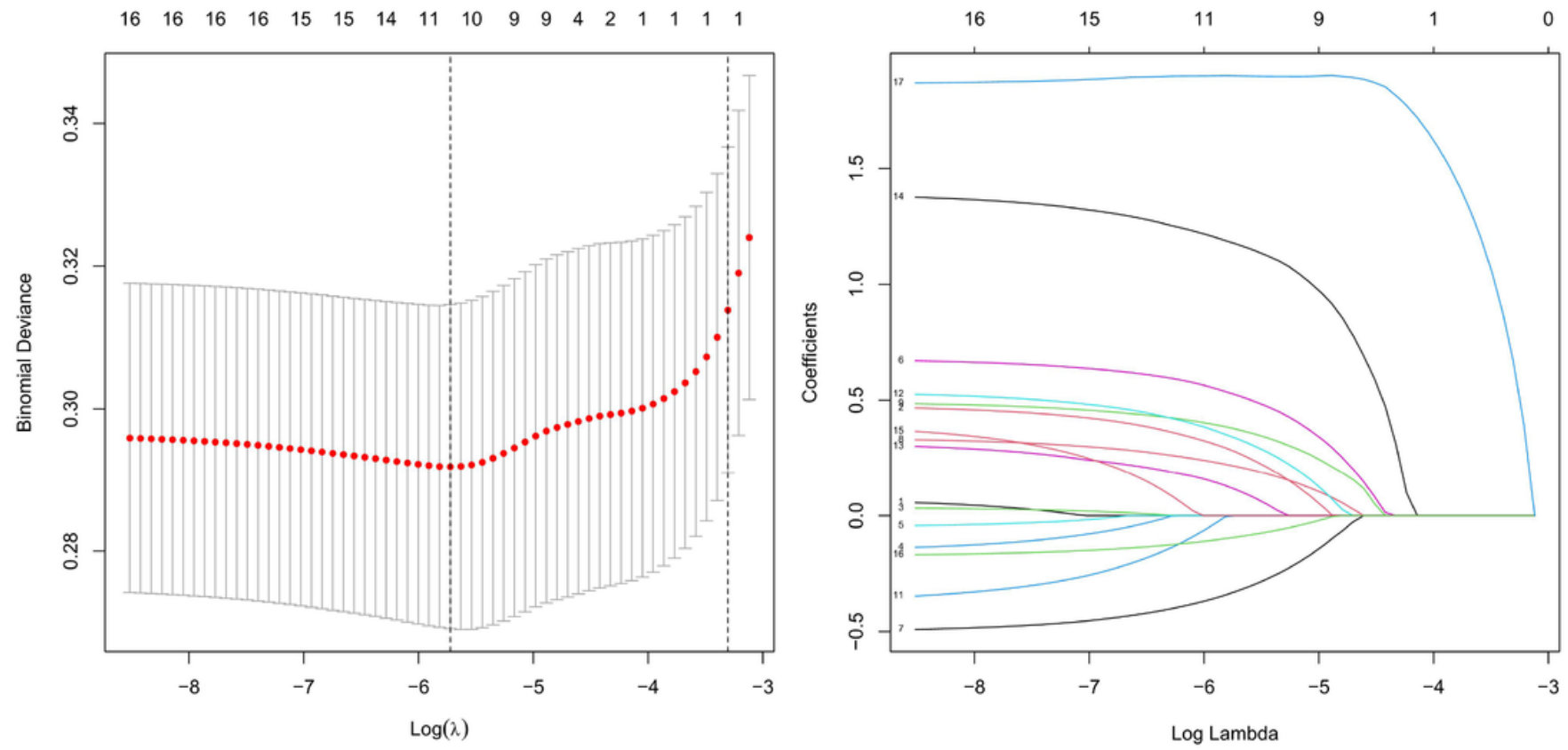

Figure 1

Demographic and clinical feature selection using the LASSO binary logistic regression model. 
Variabel Prediction Model

B OR $495 \% \mathrm{Cl}$ P value

Age

\begin{tabular}{llll}
\hline 65 & Ref & Ref & Ref \\
\hline $\mathbf{6 5}$ & 0.521 & $1.684(1.056,2.686)$ & 0.029
\end{tabular}

ASA_Status

\begin{tabular}{llll}
$\mathbf{1}$ & Ref & Ref & Ref \\
\hline $\mathbf{2}$ & 0.293 & $1.341(0.468,3.839)$ & 0.585 \\
\hline $\mathbf{3}$ & 1.167 & $3.212(0.994,10.377)$ & 0.051
\end{tabular}

\section{Type_of_Anaesthesia}

$\begin{array}{llll}\text { GA } & \text { Ref } & \text { Ref } & \text { Ref } \\ \text { RA } & -0.464 & 0.629(0.407,0.971) & 0.037\end{array}$

Operation_Duration

$\begin{array}{llll}\mathbf{0 1 0 0} & \text { Ref } & \text { Ref } & \text { Ref } \\ >\mathbf{> 1 0 0} & 0.346 & 1.414(0.863,2.316) & 0.169\end{array}$

Procedure_Description

\begin{tabular}{llll} 
Unilateral & Ref & Ref & Ref \\
\hline Bilateral & 0.669 & $1.952(1.019,3.741)$ & 0.044 \\
\hline Revision & 0.917 & $2.501(0.53,11.811)$ & 0.247 \\
\hline
\end{tabular}

\section{DM}

\begin{tabular}{llll} 
No & Ref & Ref & Ref \\
\hline Yes & 0.590 & $1.803(1.109,2.933)$ & 0.017
\end{tabular}

IHD

$\begin{array}{llll}\text { No } & \text { Ref } & \text { Ref } & \text { Ref } \\ \text { Yes } & 0.319 & 1.376(0.626,3.025) & 0.427\end{array}$

CHF

\begin{tabular}{llll} 
No & Ref & Ref & Ref \\
\hline Yes & 1.322 & $3.75(1.157,12.156)$ & 0.028 \\
\hline
\end{tabular}

Day_of_operation 


\begin{tabular}{llll} 
Mon & Ref & Ref & Ref \\
\hline Tue & -0.072 & $0.931(0.513,1.689)$ & 0.813 \\
\hline Wed & -0.756 & $0.470(0.227,0.971)$ & 0.041 \\
\hline Thu & -1.327 & $0.265(0.122,0.579)$ & 0.001 \\
\hline Fri & -0.112 & $0.894(0.476,1.680)$ & 0.729 \\
\hline Sat & -1.212 & $0.298(0.067,1.317)$ & 0.110
\end{tabular}

\section{blood_transfusion}

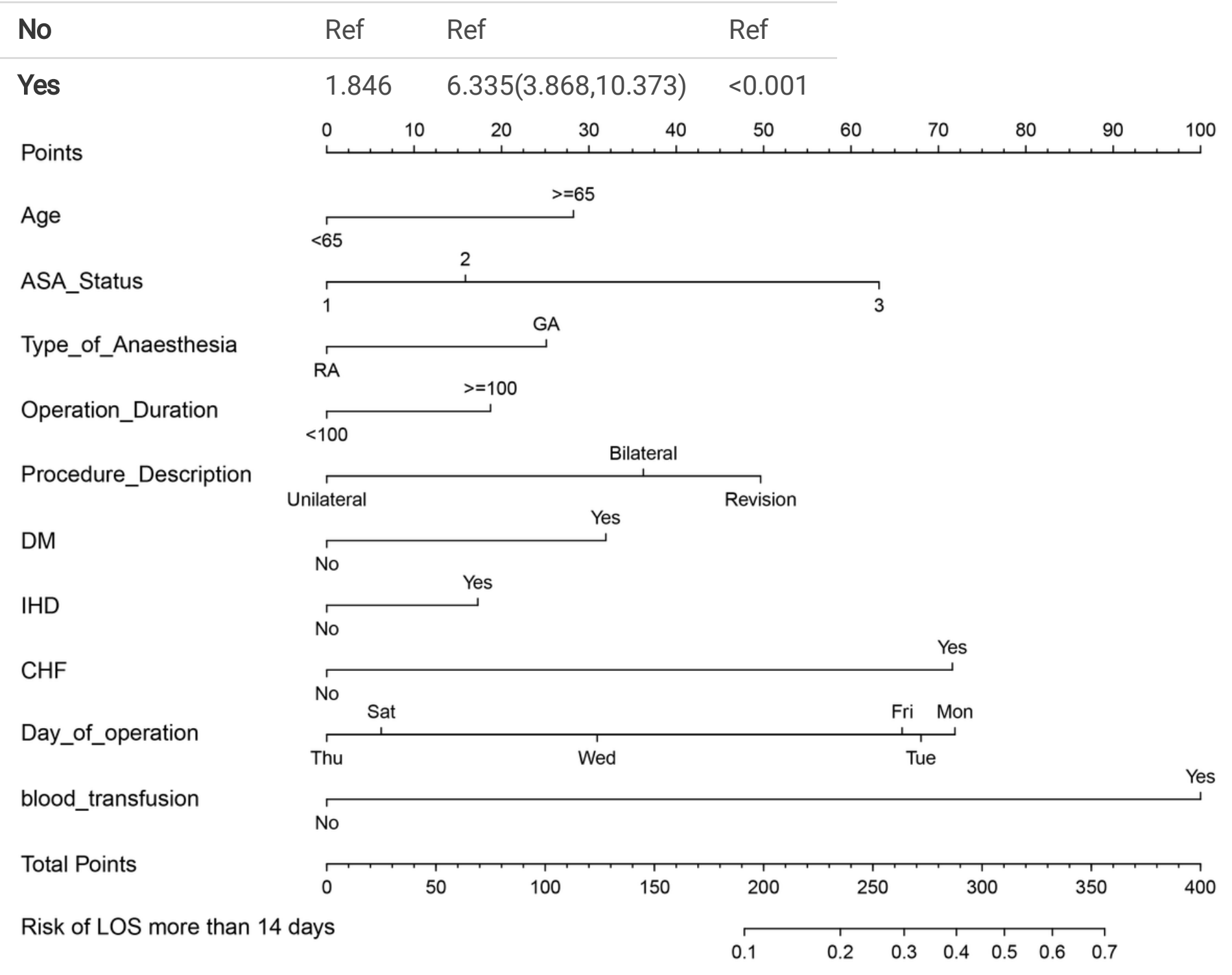

\section{Figure 2}

The Risk Nomogram for LOS More Than 14 days in Undergoing TKA Patients. 
$A \cup C=0.797$

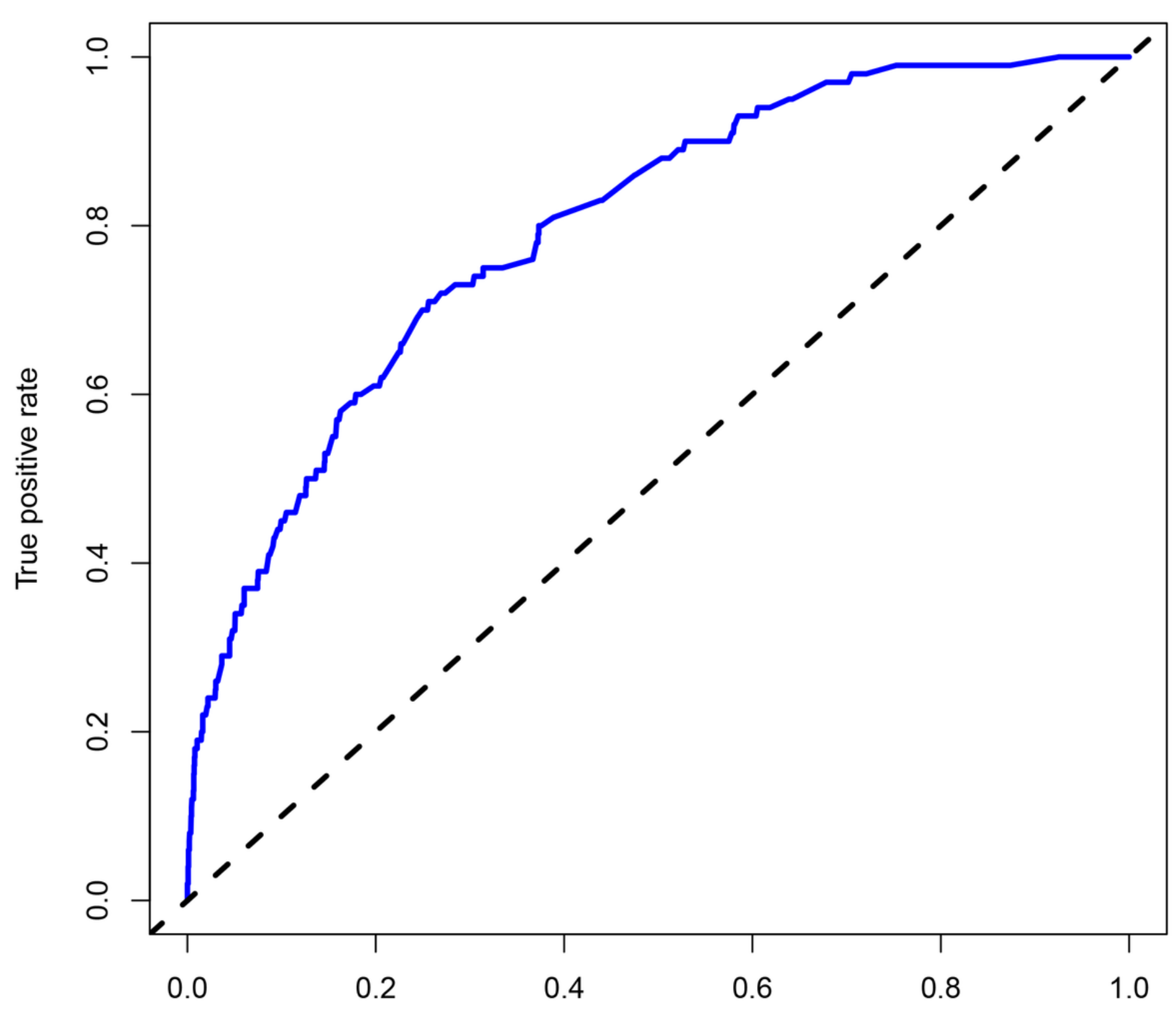

False positive rate

Figure 3

The ROC curves of the LOS more than 14 days in Undergoing TKA Patients.

Page 17/19 


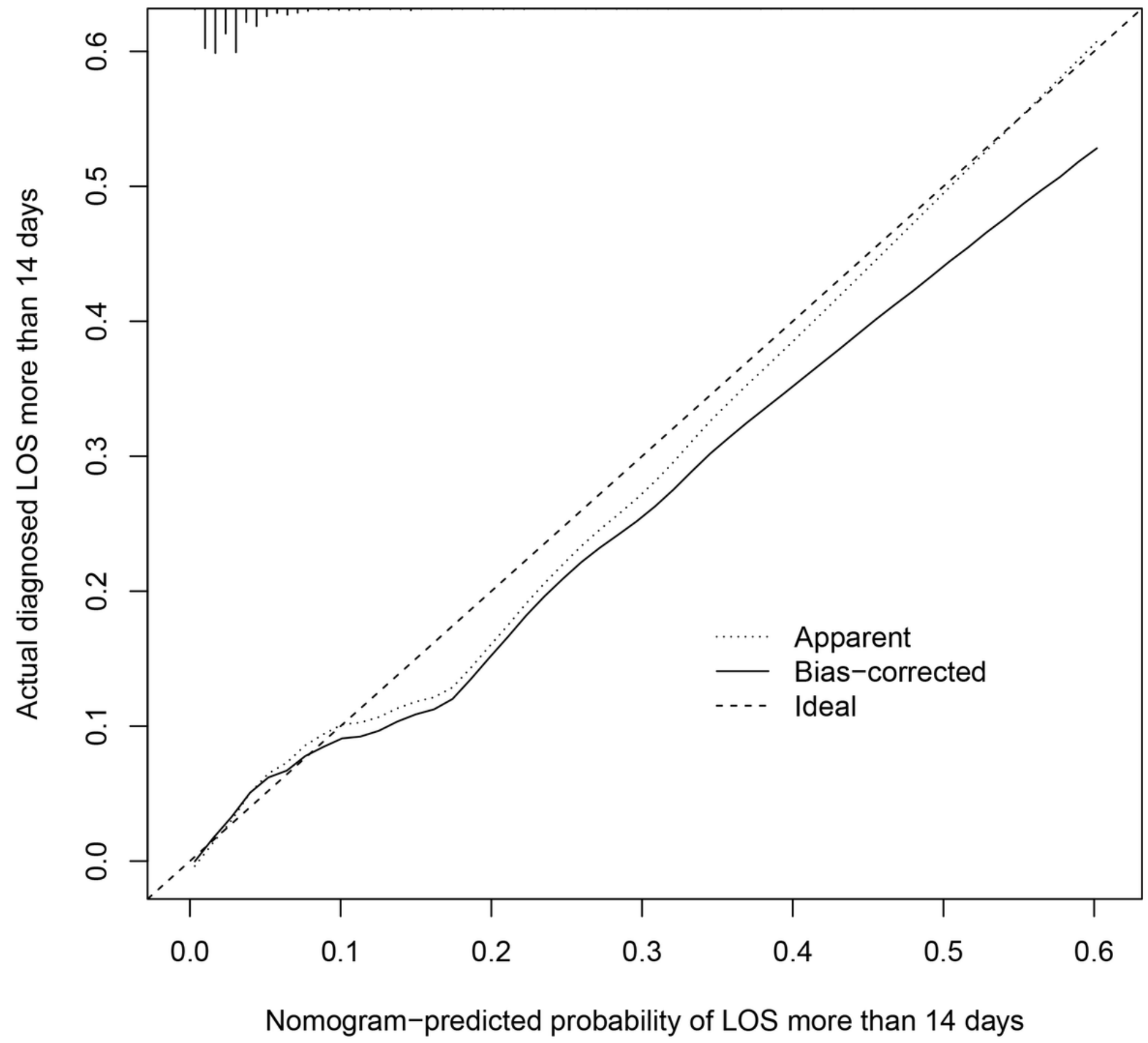

Figure 4

The Calibration curves of LOS More Than 14 days in Undergoing TKA Patients. 


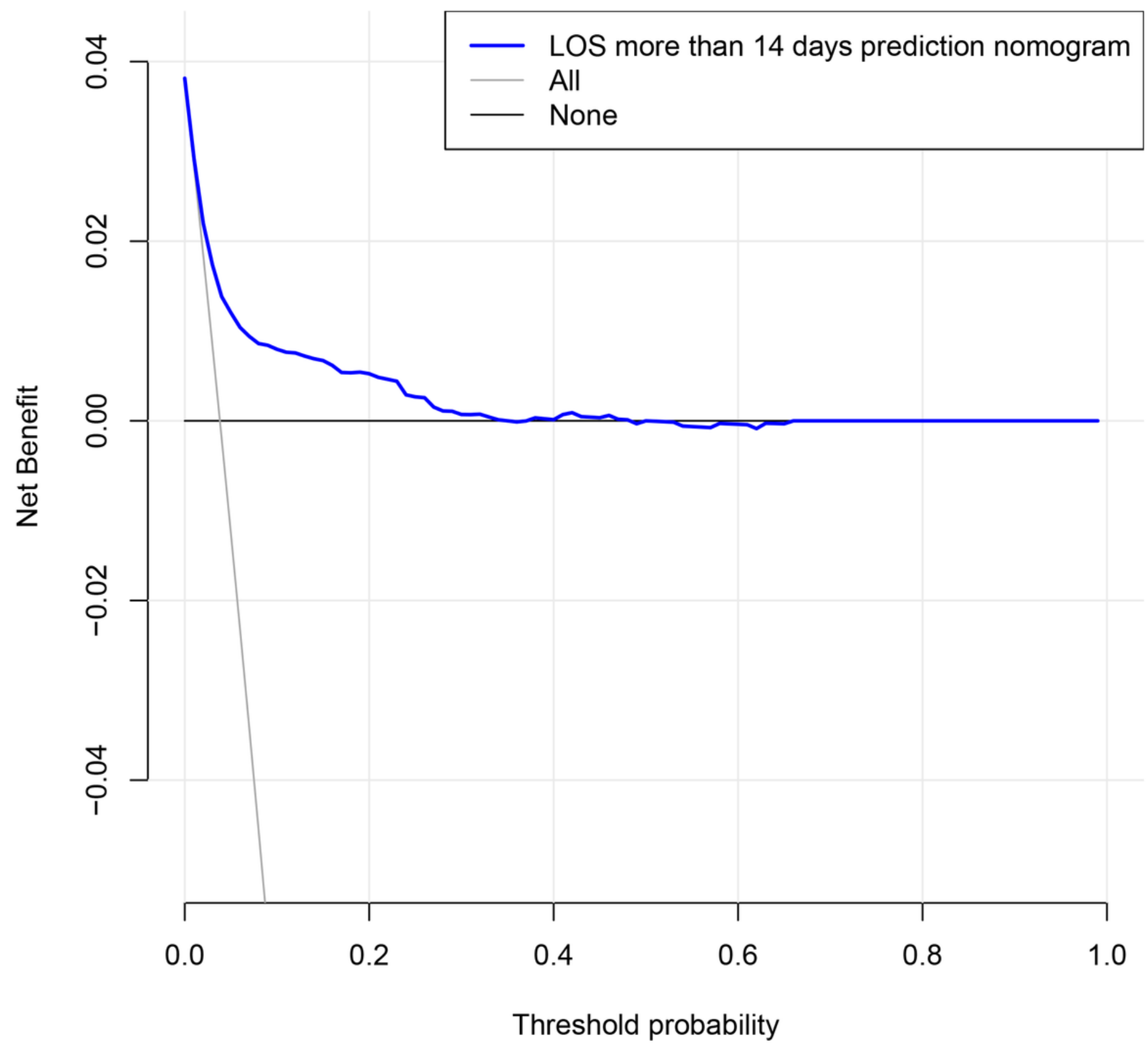

Figure 5

The Decision curve of LOS More Than 14 days in Undergoing TKA Patients. 